\title{
THE MOMENT MAP OF A LIE GROUP REPRESENTATION
}

\author{
N. J. WILDBERGER
}

\begin{abstract}
The classical moment map of symplectic geometry is used to canonically associate to a unitary representation of a Lie group $G$ a $G$-invariant subset of the dual of the Lie algebra. This correspondence is in some sense dual to geometric quantization. The nature and convexity of this subset is investigated for $G$ compact semisimple.
\end{abstract}

\section{INTRODUCTION}

Since Kirillov's fundamental paper [7], orbit theory has played an important role in the representation theory of Lie groups. The general philosophy is that there should be some connection between irreducible unitary representations of a Lie group $G$ and orbits of the group in the dual $\mathscr{G}^{*}$ of its Lie algebra $\mathscr{G}$. This connection should be functorial; the usual operations of restriction, induction, taking direct sums and tensor products of representations should correspond to geometric relationships between the associated coadjoint orbits. Properties of a representation such as square-integrability, temperedness etc. should be related to geometric aspects of the associated orbit.

Kirillov's original theory showed that if $G$ was a connected simply connected nilpotent Lie group one could establish such a bijection between the set of coadjoint orbits and the set of equivalence classes of irreducible unitary representations $\widehat{G}$ by associating to each orbit a certain induced representation. Kostant [9] noticed the close similarity between this association and what physicists refer to as quantization of a classical mechanical system, and he and Souriau [11] independently generalized this procedure to what is called geometric quantization. This theory allows one to associate representations to many families of coadjoint orbits of certain Lie groups. For example if $G$ is compact semisimple, this theory establishes the bijection between integral coadjoint orbits and $\widehat{G}$ given by the Borel-Weil theory. For a general Lie group however the geometric quantization program runs into serious difficulties and indeed the exact relationship between representations and coadjoint orbits is somewhat mysterious.

In this paper we associate to any finite-dimensional unitary representation $(V, \rho)$ of a Lie group $G$ a certain subset $I_{\rho}$ of $\mathscr{G}^{*}$ which we call the moment set of the representation. This set is the image of the moment map associated to

Received by the editors December 20, 1989.

1980 Mathematics Subject Classification (1985 Revision). Primary 22E46; Secondary 53C57.

Key words and phrases. Moment map, unitary representations, coadjoint orbits, convexity.

This research was supported by NSERC grant 0GP0007918. 
the Hamiltonian action of $G$ on the projective space $P V$. Moment maps and their images have recently been studied by a number of authors (Guillemin and Sternberg [4, 5], Atiyah [2, 3], Kirwan [8]). Of special interest is the question of convexity of the images.

In $\S 1$ we make our definitions and derive an explicit formula for the moment map of a representation, which in turn allows the definition of a moment set $I_{\rho}$ for $\rho$ an infinite-dimensional representation. In $\S 2$ some basic functorial aspects of the association $\rho \rightarrow I_{\rho}$ are studied. These are analogous to the results of Kirillov for nilpotent groups. In $\S 3$ we investigate moment sets of irreducible representations of compact semisimple groups. Theorem 3.4 shows that the extremal points of $I_{\rho}$ form a single coadjoint orbit in $\mathscr{G}^{*}$ which is the orbit of the highest weight $\lambda$.

Define a weight $\lambda$ to be root distinct if $\{\lambda-s \cdot \lambda \mid s \in W\}$ contains no roots, where $W$ is the Weyl group. The main result is Theorem 3.6 which asserts that $I_{\rho}$ is convex if and only if $\lambda$ is root distinct.

In $\S 4$ we discuss some examples.

\section{1}

Let $G$ be a connected Lie group with Lie algebra $\mathscr{G}$ and let $\mathscr{G}^{*}$ be the linear dual of $\mathscr{G}$. Let $(M, \omega)$ be a symplectic manifold and suppose that $G$ acts as a group of symplectomorphisms of $M$. Recall that this action is termed Hamiltonian if for each $X \in \mathscr{G}$ there is a real-valued smooth function $H_{X}$ on $M$ such that

(i) if $\nu_{X}$ is the vector field on $M$ generated by $X$, then $d H_{X}(\eta)=$ $\omega\left(\nu_{X}, \eta\right)$ for all vector fields $\eta$ on $M$, and

(ii) the map $X \rightarrow H_{X}$ is a Lie algebra homomorphism from $\mathscr{G}$ to the Lie algebra of smooth functions on $M$ under the Poisson bracket.

In such a case one defines the moment map $\phi: M \rightarrow \mathscr{G}^{*}$ by $\phi(m)(X)=H_{X}(m)$ for $m \in M$ and $X \in \mathscr{G}$.

Now suppose that $(V, \rho)$ is a finite-dimensional unitary representation of $G$. Let $P V$ be the projective space of all complex lines in $V$. Then $P V$ is a symplectic manifold with 2-form $\omega$ which may be described as follows (see Arnold [1]). Let $\pi: V \backslash 0 \rightarrow P V$ denote the mapping taking a point $v \neq 0$ of $V$ to the line $[v]$ passing through $v$. Let $\zeta_{1}$ and $\zeta_{2}$ be two vectors in the tangent space to $P V$ at $[v]$. Then we may find two vectors $\xi_{1}$ and $\xi_{2}$ in the tangent space to $V$ at $v, T V_{v} \simeq V$, such that $d \pi\left(\xi_{i}\right)=\zeta_{i}$ for $i=1,2$. Then

$$
\omega_{[v]}\left(\zeta_{1}, \zeta_{2}\right)=2 \operatorname{Im} \frac{\left\langle\xi_{1}, \xi_{2}\right\rangle\langle v, v\rangle-\left\langle\xi_{1}, v\right\rangle\left\langle v, \xi_{2}\right\rangle}{\langle v, v\rangle^{2}} .
$$

The action of $G$ on $V$ induces an action of $G$ on $P V$ which is easily seen from (1.1) to consist of symplectomorphisms.

Proposition 1.1. The action of $G$ on $P V$ is Hamiltonian and the associated moment map is

$$
\phi([v])(X)=\frac{1}{i} \frac{\langle X \cdot v, v\rangle}{\langle v, v\rangle},
$$

for $[v] \in P V$ and $X \in \mathscr{G}$. 
Proof. For $X \in \mathscr{G}$, let $H_{X}$ be the function on $P V$ defined by

$$
H_{X}([v])=\frac{1}{i} \frac{\langle X \cdot v, v\rangle}{\langle v, v\rangle} .
$$

For $\zeta \in T(P V)_{[v]}$, let $\xi \in T V_{v}$ such that $d \pi(\xi)=\zeta$. Then

$$
\begin{aligned}
d H_{X}(\zeta) & =\left.\frac{1}{i} \frac{d}{d t} \frac{\langle X \cdot(v+t \xi), v+t \xi\rangle}{\langle v+t \xi, v+t \xi\rangle}\right|_{t=0} \\
& =\frac{1}{i} \frac{(\langle X \cdot v, \xi\rangle+\langle X \cdot \xi, v\rangle)\langle v, v\rangle-\langle X \cdot v, v\rangle(\langle v, \xi\rangle+\langle\xi, v\rangle)}{\langle v, v\rangle^{2}} \\
& =\frac{2 \operatorname{Im}\langle X \cdot v, \xi\rangle\langle v, v\rangle+2 i\langle X \cdot v, v\rangle \operatorname{Re}\langle v, \xi\rangle}{\langle v, v\rangle^{2}} \\
& =\omega_{[v]}\left(\nu_{X}([v]), \zeta\right) .
\end{aligned}
$$

Thus condition (i) above is satisfied. Note that we have used the fact that $X$ acts as a skew-Hermitian operator on $V$. To check condition (ii), let $X, Y \in$ $\mathscr{G}$. Then the Poisson bracket of $H_{X}$ and $H_{Y}$ is defined to be $\left\{H_{X}, H_{Y}\right\}=$ $\omega\left(\nu_{X}, \nu_{Y}\right)$. Thus for $[v] \in P V$,

$$
\begin{aligned}
\left\{H_{X}, H_{Y}\right\}([v]) & =2 \operatorname{Im} \frac{\langle X \cdot v, Y \cdot v\rangle\langle v, v\rangle-\langle X \cdot v, v\rangle\langle v, Y \cdot v\rangle}{\langle v, v\rangle^{2}} \\
& =2 \operatorname{Im} \frac{\langle X \cdot v, Y \cdot v\rangle}{\langle v, v\rangle} \\
& =\frac{1}{i} \frac{\langle X \cdot v, Y \cdot v\rangle-\langle Y \cdot v, X \cdot v\rangle}{\langle v, v\rangle} \\
& =\frac{1}{i} \frac{\langle[X, Y] \cdot v, v\rangle}{\langle v, v\rangle} \\
& =H_{[X, Y]}([v]),
\end{aligned}
$$

as required.

Definition 1.2. The map $\phi: P V \rightarrow \mathscr{G}^{*}$ in Proposition 1.1 will be called the moment map of the representation $\rho$.

Now the group $G$ acts on $\mathscr{G}^{*}$ by the coadjoint action and it is readily seen that $\phi$ commutes with the actions of $G$ on $P V$ and $\mathscr{G}^{*}$. The image of $\phi$ will thus be a $G$-invariant subset of $\mathscr{G}^{*}$, necessarily compact since we have assumed $V$ finite dimensional.

Definition 1.3. The image of $\phi$

$$
I_{\rho}=\{\phi([v]) \mid[v] \in P V\},
$$

will be called the moment set of the representation $\rho$.

We will call a representation $(V, \rho)$ trivial if $\rho(g)$ is the identity operator for all $g \in G$. Since $G$ is connected, this is equivalent to the condition that $\rho(X)=0$ for all $X \in \mathscr{G}$ :

Lemma 1.4. The representation $(V, \rho)$ is trivial if and only if $I_{\rho}=\{0\}$.

Proof. Clearly if $(V, \rho)$ is trivial, then $I_{\rho}=\{0\}$ and conversely if $I_{\rho}=\{0\}$ then for all $X \in \mathscr{G}$ and all $v \in V$,

$$
\langle X \cdot v, v\rangle=0 .
$$


But it is an elementary fact that if $V$ is a complex vector space then this implies that $X \cdot v=0$ for all $v \in V$.

Corollary 1.5. Suppose $G$ is a Lie group with the property that the only compact G-invariant subset of $\mathscr{G}^{*}$ is $\{0\}$. Then any finite-dimensional unitary representation of $G$ is trivial.

Proof. This is immediate from the previous lemma and the remarks preceding it.

We remark that Corollary 1.5 presents a new proof of the fact that $\operatorname{SL}(2, \mathbb{R})$ (for example) has no nontrivial finite-dimensional unitary representations.

Definition 1.6. A representation $(V, \rho)$ will be called convex if $I_{\rho}$ is convex.

If $(V, \rho)$ is an infinite-dimensional unitary representation of $G$, let $V^{\infty}$ denote the space of $C^{\infty}$ vectors of $\rho$. Then for each $v \in V^{\infty}$, we define $\Psi(v) \in \mathscr{G}^{*}$ by

$$
\Psi(v)(X)=\frac{1}{i} \frac{\langle X \cdot v, v\rangle}{\langle v, v\rangle},
$$

and define the moment set $I_{\rho}$ to be

$$
I_{\rho}=\operatorname{cl}\left\{\Psi(v) \mid v \in V^{\infty}\right\} .
$$

In this paper we deal primarily with the finite-dimensional case.

In this section some functorial aspects of the correspondence between representations $\rho$ and moment sets $I_{\rho}$ are established. It will be useful to first establish some more notation. Let $\Omega=\{v \in V \mid\langle v, v\rangle=1\}$ be the unit spheres of $V$ and denote by $\pi$ the mapping from $\Omega$ to $P V$ sending $v$ to $[v]$, the so-called Hopf fibration. It exhibits $\Omega$ as an $S^{1}$-principal bundle over $P V$, with the action of $S^{1}$ on a fibre being just multiplication by $e^{i \theta}$ in the vector space $V$. Let $\Psi: \Omega \rightarrow \mathscr{G}^{*}$ be the lift $\Psi=\phi \circ \pi$ so that for $v \in \Omega$ and $X \in \mathscr{G}$,

$$
\Psi(v)(X)=\frac{1}{i}\langle X \cdot v, v\rangle \quad \text { and } \quad I_{\rho}=\{\Psi(v) \mid v \in \Omega\} .
$$

Lemma 2.1. Let $\left(V_{1}, \rho_{1}\right)$ and $\left(V_{2}, \rho_{2}\right)$ be two unitary representations of $G$ and let $V=V_{1} \oplus V_{2}$ and $\rho=\rho_{1} \oplus \rho_{2}$. Then

$$
I_{\rho}=\left\{f \in \mathscr{G}^{*} \mid f=t f_{1}+(1-t) f_{2}, 0 \leq t \leq 1 \text { and } f_{i} \in I_{\rho_{i}}, \quad i=1,2\right\} \text {. }
$$

Proof. Let $\Omega_{1}, \Omega_{2}$ and $\Omega$ be the unit spheres of $V_{1}, V_{2}$ and $V$ respectively. Then for $v \in \Omega$ we can write $v=z_{1} v_{1}+z_{2} v_{2}$ with $v_{i} \in \Omega_{i}, i=1,2$, and $z_{i} \in \mathbb{C}$ satisfying $\left|z_{1}\right|^{2}+\left|z_{2}\right|^{2}=1$. Let $\Psi_{1}, \Psi_{2}$ and $\Psi$ be the lifted moment maps of $\rho_{1}, \rho_{2}$ and $\rho$ respectively. Then for $X \in \mathscr{G}$,

$$
\begin{aligned}
\Psi(v)(X) & =\frac{1}{i}\left\langle X \cdot\left(z_{1} v_{1}+z_{2} v_{2}\right), z_{1} v_{1}+z_{2} v_{2}\right\rangle \\
& =\left(\left|z_{1}\right|^{2} \Psi_{1}\left(v_{1}\right)+\left|z_{2}\right|^{2} \Psi_{2}\left(v_{2}\right)\right)(X),
\end{aligned}
$$

since $\left\langle X \cdot v_{1}, v_{2}\right\rangle=\left\langle X \cdot v_{2}, v_{1}\right\rangle=0$. Combining (2.1) with (2.2) we get the result. 
Lemma 2.2. Let $(V, \rho)$ be a unitary representation of $G$ and let $H$ be a Lie

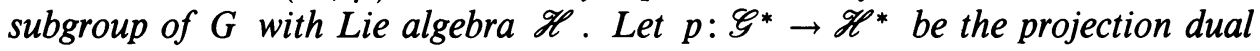
to the inclusion $i: \mathscr{H} \rightarrow \mathscr{G}$. Then

$$
I_{\left.\rho\right|_{H}}=p\left(I_{\rho}\right) .
$$

Proof. Obvious from the definitions.

Proposition 2.3. Let $\left(V_{1}, \rho_{1}\right)$ and $\left(V_{2}, \rho_{2}\right)$ be two unitary representations of Lie groups $G_{1}$ and $G_{2}$ respectively. Let $V=V_{1} \otimes V_{2}$ and let $\rho=\rho_{1} \otimes \rho_{2}$ be the representation of $G=G_{1} \times G_{2}$ given by $\rho\left(g_{1}, g_{2}\right) v_{1} \otimes v_{2}=\rho_{1}\left(g_{1}\right) v_{1} \otimes \rho_{2}\left(g_{2}\right) v_{2}$ for $g_{i} \in G_{i}$ and $v_{i} \in V_{i}, i=1,2$. Then

$$
I_{\rho_{1}} \times I_{\rho_{2}} \subseteq I_{\rho} \subseteq \operatorname{conv}\left(I_{\rho_{1}}\right) \times \operatorname{conv}\left(I_{\rho_{2}}\right)
$$

where we identify $\mathscr{G}^{*}$ with $\mathscr{G}_{1}^{*} \times \mathscr{G}_{2}^{*}$ and where $\operatorname{conv}(S)$ denotes the convex hull of the set $S$.

Proof. Let $\Omega_{1}, \Omega_{2}$ and $\Omega$ be the unit spheres of $V_{1}, V_{2}$ and $V$ respectively and let $\Psi_{1}, \Psi_{2}$ and $\Psi$ be the lifted moment maps of $\rho_{1}, \rho_{2}$ and $\rho$ respectively. Then for any $v_{i} \in \Omega_{i}, i=1,2$, we have $v=v_{1} \otimes v_{2} \in \Omega$ so that for $X=\left(X_{1}, X_{2}\right) \in \mathscr{G}$,

$$
\begin{aligned}
\Psi(v)(X) & =\frac{1}{i}\left\langle X_{1} \cdot v_{1} \otimes v_{2}+v_{1} \otimes X_{2} \cdot v_{2}, v_{1} \otimes v_{2}\right\rangle \\
& =\Psi_{1}\left(v_{1}\right)\left(X_{1}\right)+\Psi_{2}\left(v_{2}\right)\left(X_{2}\right) .
\end{aligned}
$$

Thus

$$
I_{\rho_{1}} \times I_{\rho_{2}} \subseteq I_{\rho}
$$

Now let $\left\{e_{1}, \ldots, e_{m}\right\}$ be an orthonormal basis of $V_{1}$. Then if $v \in \Omega$ we may write

$$
v=\sum_{k=1}^{m} e_{k} \otimes w_{k}
$$

with $w_{k} \in V_{2}$ for $k=1, \ldots, m$ and $\sum_{k=1}^{m}\left|w_{k}\right|^{2}=1$. For $X=\left(X_{1}, X_{2}\right) \in \mathscr{G}$,

$$
\Psi(v)(X)=\frac{1}{i} \sum_{k, l=1}^{m}\left\langle X_{1} \cdot e_{k} \otimes w_{k}+e_{k} \otimes X_{2} \cdot w_{k}, e_{l} \otimes w_{l}\right\rangle,
$$

so that

$$
\begin{aligned}
\Psi(v)\left(0, X_{2}\right) & =\frac{1}{i} \sum_{k, l=1}^{m} \delta_{k l}\left\langle X_{2} \cdot w_{k}, w_{l}\right\rangle \\
& =\left(\sum_{\substack{k=1 \\
\left|w_{k}\right| \neq 0}}^{m}\left|w_{k}\right|^{2} \Psi_{2}\left(u_{k}\right)\right)\left(X_{2}\right),
\end{aligned}
$$

where $u_{k}=w_{k} /\left|w_{k}\right|$ if $\left|w_{k}\right| \neq 0$. Thus if $p_{2}: \mathscr{G}_{1}^{*} \times \mathscr{G}_{2}^{*} \rightarrow \mathscr{G}_{2}^{*}$ is the projection onto the second factor, we see that

$$
p_{2}\left(I_{\rho}\right) \subseteq \operatorname{conv}\left(I_{\rho_{2}}\right)
$$

so that by symmetry

$$
I_{\rho} \subseteq \operatorname{conv}\left(I_{\rho_{1}}\right) \times \operatorname{conv}\left(I_{\rho_{2}}\right)
$$


Lemma 2.4. Let $\left(V_{1}, \rho_{1}\right)$ and $\left(V_{2}, \rho_{2}\right)$ be two unitary representations of $G$. Let $V=V_{1} \otimes V_{2}$ and let $\rho$ be the representation of $G$ on $V$ given by $\rho(g) v_{1} \otimes v_{2}=$ $\rho_{1}(g) v_{1} \otimes \rho_{2}(g) v_{2}$ for all $v_{i} \in V_{i}, i=1,2$. Then

$$
I_{\rho_{1}}+I_{\rho_{2}} \subseteq I_{\rho} \subseteq \operatorname{conv}\left(I_{\rho_{1}}\right)+\operatorname{conv}\left(I_{\rho_{2}}\right) \text {. }
$$

Proof. This follows from the previous two lemmas by considering $G \subseteq G \times G$ as the diagonal subgroup of elements $(g, g), g \in G$.

In this section we assume that $G$ is compact. We wish to study the nature of the moment sets of representations. The simplest case is $G$ abelian. The following result is a special case of a general convexity result (see Atiyah [2]; Guillemin and Sternberg [4]).

Lemma 3.1. Let $T$ be a torus with Lie algebra $\mathscr{T}$. Let $(V, \rho)$ be a finite dimensional unitary representation of $T$ with weights $i \lambda_{1}, \ldots, i \lambda_{n} \in i \mathscr{T}^{*}$. Then $I_{\rho}$ is the convex hull of $\left\{\lambda_{1}, \ldots, \lambda_{n}\right\}$ in $\mathscr{T}^{*}$. In particular $I_{\rho}$ is convex.

Proof. Write $V=V_{1} \oplus \cdots \oplus V_{n}$ with $\left(V_{j}, \rho_{j}\right)$ the 1-dimensional representation with weight $i \lambda_{j}$, that is, for $X \in \mathscr{T}$ and $v_{j} \in V_{j}$,

$$
X \cdot v_{j}=i \lambda_{j}(X) v_{j}
$$

Then clearly $I_{\rho_{j}}=\left\{\lambda_{j}\right\}$, so the result follows from Lemma 2.1 by induction.

Returning to the general case of $G$ compact, choose a $G$-invariant negativedefinite form $($,$) on \mathscr{G}$, so that $\mathscr{G}$ and $\mathscr{G}^{*}$ may be identified. Let $T \subseteq G$ be a maximal torus with Lie algebra $\mathscr{T}$ and let $W$ be its Weyl group. We will use the form $($,$) to consider \mathscr{T}^{*} \subseteq \mathscr{G}^{*}$, with $p: \mathscr{G}^{*} \rightarrow \mathscr{T}^{*}$ the orthogonal projection.

For any coadjoint orbit $\mathscr{O} \subseteq \mathscr{G}^{*}, \mathscr{O} \cap \mathscr{T}^{*}$ is a $W$-orbit and a well-known result of Kostant [10] states that

$$
p(\mathscr{O})=\operatorname{conv}\left\{\mathscr{O} \cap \mathscr{T}^{*}\right\} .
$$

Let $(V, \rho)$ be any finite-dimensional unitary representation of $G$ and let its weights with respect to $\mathscr{T}$ be $i \lambda_{1}, \ldots, i \lambda_{n} \in i \mathscr{T}^{*}$ with corresponding weight vectors $v_{1}, \ldots, v_{n} \in \Omega$. Let

$$
D=\operatorname{conv}\left\{\lambda_{1}, \ldots, \lambda_{n}\right\}
$$

and let $D^{G}=G \cdot D \subseteq \mathscr{G}^{*}$.

Proposition 3.2. $I_{\rho} \subseteq D^{G}$.

Proof. From Lemmas 2.2 and 3.1 we have

$$
p\left(I_{\rho}\right)=I_{\left.\rho\right|_{T}}=D \text {. }
$$

Thus

$$
I_{\rho} \cap \mathscr{T}^{*} \subseteq p\left(I_{\rho}\right) \subseteq D .
$$

Now $D$ is a $W$-invariant set, so that $D^{G} \cap \mathscr{T}^{*}=D$. Thus $I_{\rho} \cap \mathscr{T}^{*} \subseteq D^{G} \cap \mathscr{T}^{*}$ so that $I_{\rho} \subseteq D^{G}$.

For any convex set $S \subseteq \mathscr{G}^{*}$, let $\operatorname{Ext}(S)$ denote the extremal set of $S$, that is, those elements of $S$ not properly contained in any open line segment in $S$. 
Lemma 3.3. If $\omega_{k} \in \Omega$ is a weight vector of weight $i \lambda_{k}$ then $\Psi\left(\omega_{k}\right)=\lambda_{k}$. Conversely if $\lambda_{k} \in \operatorname{Ext}(D)$ and $w \in \Omega$ with $\Psi(w)=\lambda_{k}$, then $w$ is a weight vector of weight $i \lambda_{k}$.

Proof. If $\omega_{k}$ is a weight vector then $\Psi\left(\omega_{k}\right)$ is fixed by $T$ and thus in $\mathscr{T}^{*}$, which implies that actually $\Psi\left(\omega_{k}\right)=\lambda_{k}$.

If $w \in \Omega$ with $\Psi(\omega)=\lambda_{k} \in \operatorname{Ext}(D)$, write

$$
w=\sum_{j=1}^{n} z_{j} v_{j}
$$

with $z_{j} \in \mathbb{C}$ and $\sum_{j=1}^{n}\left|z_{j}\right|^{2}=1$. Then for any $X \in \mathscr{T}$,

$$
\Psi(\omega)(X)=\frac{1}{i} \sum_{j=1}^{n}\left|z_{j}\right|^{2}\left\langle X \cdot v_{j}, v_{j}\right\rangle=\left(\sum_{j=1}^{n}\left|z_{j}\right|^{2} \lambda_{j}\right)(X) .
$$

Thus $\lambda_{k}=\sum_{j=1}^{n}\left|z_{j}\right|^{2} \lambda_{j}$. Since $\lambda_{k} \in \operatorname{Ext}(D)$, all the $\lambda_{j}$ that appear in this sum must actually be equal to $\lambda_{k}$, so that $\omega$ is a sum of weight vectors of weight $i \lambda_{k}$ and is thus itself a weight vector of weight $i \lambda_{k}$.

Theorem 3.4. Let $G$ be a semisimple compact Lie group and $(V, \rho)$ an irreducible unitary representation of $G$. Then $\operatorname{Ext}\left(\operatorname{conv}\left(I_{\rho}\right)\right)$ is a single coadjoint orbit in $\mathscr{G}^{*}$.

Proof. In this case it is well known that the weights $i \lambda_{1}, \ldots, i \lambda_{n}$ of $(V, \rho)$ are contained in the convex hull of the $W$ orbit of one of them, say $i \lambda_{1}$ (see Helgason [6]). If $\mathscr{O}$ is the coadjoint orbit through $\lambda_{1}$, then $\mathscr{O}$ intersects $\mathscr{T}^{*}$ in exactly $\operatorname{Ext}(D)$, the set of $W$-orbits of $\lambda_{1}$. Now since $\operatorname{Ext}\left(\operatorname{conv}\left(I_{\rho}\right)\right) \cap \mathscr{T}^{*}=$ $\operatorname{Ext}(D)=\mathscr{O} \cap \mathscr{T}^{*}$ we conclude that $\operatorname{Ext}\left(\operatorname{conv}\left(I_{\rho}\right)\right)=\mathscr{O}$.

Let $\mathscr{G}_{\mathbb{C}}$ be the complexification of $\mathscr{G}$ and let $\mathscr{H}$ be the Cartan subalgebra of $\mathscr{G}_{\mathbb{C}}$ containing $\mathscr{T}$. Let $\mathscr{H}^{*}$ denote the complex-linear dual of $\mathscr{H}$ and let

$$
\mathscr{H}_{0}^{*}=\left\{f \in \mathscr{H}^{*} \mid f(X) \in i \mathbb{R} \forall X \in \mathscr{T}\right\} .
$$

The form ( , ) on $\mathscr{G}$ can be considered to be the restriction to $\mathscr{G}$ of the Killing form on $\mathscr{G}_{\mathbb{C}}$, which gives an inner product on $\mathscr{H}_{0}^{*}$.

Let $\Sigma \subseteq \mathscr{H}_{0}^{*}$ denote the set of roots of $\mathscr{G}_{\mathbb{C}}$ so that

$$
\mathscr{G}_{\mathbb{C}}=\mathscr{H} \oplus \sum_{\alpha \in \Sigma} \mathscr{G}_{\alpha},
$$

where each root space $\mathscr{G}_{\alpha}$ is one-dimensional and orthogonal to $\mathscr{H}$. Let $\Pi=$ $\left\{\alpha_{1}, \ldots, \alpha_{l}\right\}$ denote a fixed set of fundamental roots, so that $\Pi$ forms a basis of $\mathscr{H}_{0}^{*}$ and any $\alpha \in \Sigma$ can be written as

$$
\alpha=m_{1} \alpha_{1}+\cdots+m_{l} \alpha_{l},
$$

where the $m_{j}$ are integers either all nonnegative or all nonpositive. The positive roots will be denoted $\Sigma^{+}$. If $(V, \rho)$ is a unitary representation of $G$, then its differential extends by complex linearity to a representation of $\mathscr{G}_{\mathbb{C}}$. The weights $\lambda$ of $(V, \rho)$ are then in $\mathscr{H}_{0}^{*}$ and are integral; that is

$$
2 \frac{\left(\lambda, \alpha_{j}\right)}{\left(\alpha_{j}, \alpha_{j}\right)}
$$


is an integer for all $\alpha_{j} \in \Pi$. If these integers are all nonnegative, $\lambda$ is called a dominant integral weight.

The Weyl group $W$ acts on $\mathscr{H}_{0}^{*}$ and is generated by the reflections $s_{\alpha}$, $\alpha \in \Sigma$, where

$$
s_{\alpha} \cdot \lambda=\lambda-2 \frac{(\lambda, \alpha)}{(\alpha, \alpha)} \alpha
$$

Definition 3.5. An element $\lambda \in \mathscr{H}_{0}^{*}$ will be called root distinct if $\{\lambda-s \cdot \lambda \mid s \in W\}$ contains no roots.

Theorem 3.6. Let $(V, \rho)$ be an irreducible unitary representation of $G$ of highest weight $\lambda \in \mathscr{H}_{0}^{*}$. Then $\rho$ is convex if and only if $\lambda$ is root distinct.

Proof. Suppose that $\lambda$ is root distinct. Let $\left\{\lambda=\lambda_{1}, \ldots, \lambda_{r}\right\}$ be the set of Weyl translates of $\lambda$ and for $j=1, \ldots, r$ let $v_{j}$ be a weight vector in $\Omega$ of weight $\lambda_{j}$. Let $D=\operatorname{conv}\left\{\lambda_{1}, \ldots, \lambda_{r}\right\}$. By Kostant's theorem, it suffices to show that $I_{\rho} \cap \mathscr{H}_{0}^{*}=i^{-1} D$. For $v \in \Omega$, extend $\Psi(v)$ to a complex linear functional on $\mathscr{G}_{\mathrm{C}}$.

Choose $\lambda^{\prime} \in D$; then it is possible to find $z_{j} \in \mathbb{C}, j=1, \ldots, r$ such that $\sum_{j=1}^{r}\left|z_{j}\right|^{2}=1$ and such that

$$
\sum_{j=1}^{r}\left|z_{j}\right|^{2} \lambda_{j}=\lambda^{\prime}
$$

It suffices to show that $v=\sum_{j=1}^{r} z_{j} v_{j}$ satisfies $\Psi(v)=i^{-1} \lambda^{\prime}$. Now if $X \in \mathscr{H}$, then

$$
\Psi(v)(X)=\frac{1}{i}\langle X \cdot v, v\rangle=\frac{1}{i} \sum_{j=1}^{r}\left|z_{j}\right|^{2} \lambda_{j}(X)=i^{-1} \lambda^{\prime}(X) .
$$

On the other hand, for $\alpha \in \Sigma$ and $0 \neq X_{\alpha} \in \mathscr{G}_{\alpha}, X_{\alpha}\left(v_{j}\right)$ will be a weight vector of weight $\lambda_{j}+\alpha$, which by assumption is none of the $\lambda_{k}, k=1, \ldots, r$. Thus $\Psi(v)\left(X_{\alpha}\right)=0$, so that $\Psi(v)=i^{-1} \lambda^{\prime}$. Thus $i^{-1} D \subseteq I_{\rho} \cap \mathscr{H}_{0}^{*}$; Proposition 3.2 implies that $I_{\rho} \cap \mathscr{X}_{0}^{*} \subseteq i^{-1} D$.

For the converse, a technical lemma is needed.

Lemma 3.7. Suppose that $\lambda \in \mathscr{H}_{0}^{*}$ is dominant integral and that $\{\lambda-s \cdot \lambda \mid s \in W\}$ contains no fundamental roots. Then $\lambda$ is root distinct.

Proof. Suppose that $\lambda \in \mathscr{H}_{0}^{*}$ is dominant integral, so that

$$
2 \frac{\left(\lambda, \alpha_{j}\right)}{\left(\alpha_{j}, \alpha_{j}\right)}=m_{j}
$$

is a nonnegative integer for all $\alpha_{j} \in \Pi$. The assumption on $\lambda$ implies that actually $m_{j} \neq 1$ for all $j=1, \ldots, l$.

Suppose that for some $s \in W, \lambda-s \cdot \lambda=\alpha$ is a root. Then since $(\lambda, \lambda)=$ $(s \lambda, s \lambda)$,

$$
2 \frac{(\lambda, \alpha)}{(\alpha, \alpha)}=1
$$

Write $\alpha$ as a sum of fundamental roots

$$
\alpha=\sum_{j=1}^{l} n_{j} \alpha_{j},
$$


with each $n_{j}$ a nonnegative integer. Then

$$
1=2 \frac{(\lambda, \alpha)}{(\alpha, \alpha)}=\sum_{j=1}^{l} n_{j} 2 \frac{\left(\lambda, \alpha_{j}\right)}{(\alpha, \alpha)}=\sum_{j=1}^{l} n_{j} m_{j} \frac{\left(\alpha_{j}, \alpha_{j}\right)}{(\alpha, \alpha)} .
$$

Now since for any 2 roots $\gamma, \gamma^{\prime} \in \Sigma$ it is well known that

$$
\frac{\left(\gamma^{\prime}, \gamma^{\prime}\right)}{(\gamma, \gamma)} \geq \frac{1}{3}
$$

we have that

$$
3 \geq \sum_{j=1}^{l} n_{j} m_{j} .
$$

At least one of the terms in this sum, say $n_{t} m_{t}$, must be nonzero. Then the fact that $m_{t} \neq 1$ implies that $n_{t}=1$ and that all the other terms must be zero. Thus

$$
2 \frac{\left(\lambda, \alpha_{t}\right)}{(\alpha, \alpha)}=1 \text {. }
$$

If $\beta, \beta^{\prime} \in \Sigma^{+}$and $\beta+\beta^{\prime} \in \Sigma$, then it is well known that

$$
2 \frac{\left(\beta, \beta^{\prime}\right)}{(\beta, \beta)} \quad \text { and } \quad 2 \frac{\left(\beta, \beta^{\prime}\right)}{\left(\beta^{\prime}, \beta^{\prime}\right)}
$$

are both integers less than 0 . Thus

$$
\begin{aligned}
\left(\beta+\beta^{\prime}, \beta+\beta^{\prime}\right) & =(\beta, \beta)+2\left(\beta, \beta^{\prime}\right)+\left(\beta^{\prime}, \beta^{\prime}\right) \\
& \leq \min \left\{(\beta, \beta),\left(\beta^{\prime}, \beta^{\prime}\right)\right\} .
\end{aligned}
$$

If $\beta \in \Sigma^{+}$, it is possible to express $\beta$ as a sum of fundamental roots $\beta=$ $\alpha_{i_{1}}+\cdots+\alpha_{i_{p}}$, such that $\alpha_{i_{1}}+\cdots+\alpha_{i_{q}} \in \Sigma^{+}$for all $1 \leq q \leq p$. Combining these two observations with the fact that the set of fundamental roots is linearly independent, one sees that $(\alpha, \alpha) \leq\left(\alpha_{t}, \alpha_{t}\right)$. Then

$$
2 \frac{\left(\lambda, \alpha_{t}\right)}{\left(\alpha_{t}, \alpha_{t}\right)} \leq 2 \frac{\left(\lambda, \alpha_{t}\right)}{(\alpha, \alpha)}
$$

so that

$$
2 \frac{\left(\lambda, \alpha_{t}\right)}{\left(\alpha_{t}, \alpha_{t}\right)}=1
$$

But this is a contradiction, and proves the lemma.

Continuing with the proof of the theorem, suppose that $\lambda=\lambda_{1}$ is not root distinct. Then by the above lemma there exists a fundamental root, say $\alpha_{1}$ such that

$$
s_{\alpha_{1}} \cdot \lambda_{1}=\lambda_{1}-\alpha_{1}=\lambda_{2} \text {. }
$$

Both $\lambda_{1}$ and $\lambda_{2}$ are vertices of $D$. Since every vertex of $D$ is of the form

$$
\lambda_{1}-\left(n_{1} \alpha_{1}+\cdots+n_{l} \alpha_{l}\right)
$$

with the $n_{j}$ nonnegative integers, a convex combination of such vertices can lie on the line segment $E$ joining $\lambda_{1}$ and $\lambda_{2}$ only if the convex combination contains only $\lambda_{1}$ and $\lambda_{2}$ themselves. Let $\lambda^{\prime}$ be the midpoint of $E$,

$$
\lambda^{\prime}=\frac{\lambda_{1}+\lambda_{2}}{2}=\lambda_{1}-\frac{\alpha_{1}}{2} \text {. }
$$


Then we claim that $i^{-1} \lambda^{\prime} \notin I_{\rho}$. Suppose otherwise, so that we could find $v^{\prime} \in \Omega$ with $\Psi\left(v^{\prime}\right)=i^{-1} \lambda^{\prime}$. Let $\left\{v_{1}, \ldots, v_{n}\right\}$ be an orthonormal basis of $V$ of weight vectors, with corresponding weights $\left\{\lambda_{1}, \ldots, \lambda_{n}\right\}$.

Write $v^{\prime}=\sum_{j=1}^{n} z_{j} v_{j}$ so that for $X \in \mathscr{H}$,

$$
\Psi\left(v^{\prime}\right)(X)=\frac{1}{i}\left(\sum_{j=1}^{n}\left|z_{j}\right|^{2} \lambda_{j}\right)(X)=i^{-1} \lambda^{\prime}(X) .
$$

Then $\lambda^{\prime}=\sum_{j=1}^{n}\left|z_{j}\right|^{2} \lambda_{j}$ so by the above remark, all terms in this sum are zero except for $j=1,2$. Thus $v^{\prime}=z_{1} v_{1}+z_{2} v_{2}$, with $\left|z_{1}\right|^{2}=\left|z_{2}\right|^{2}=\frac{1}{2}$. Now if $0 \neq X_{\alpha_{1}} \in \mathscr{G}_{\alpha_{1}}$, then by the uniqueness of the highest weight vector,

$$
X_{\alpha_{1}}\left(v_{2}\right)=y v_{1},
$$

for some $0 \neq y \in \mathbb{C}$. Then

$$
\Psi\left(v^{\prime}\right)\left(X_{\alpha_{1}}\right)=\frac{1}{i}\left\langle X_{\alpha_{1}} \cdot v^{\prime}, v^{\prime}\right\rangle=\frac{1}{i} z_{2} \bar{z}_{1} y \neq 0 .
$$

But $\lambda^{\prime}\left(X_{\alpha_{1}}\right)=0$ so we get a contradiction. So $i^{-1} \lambda^{\prime} \notin I_{\rho}$ and therefore $\rho$ is not convex.

In this section we discuss some examples.

Let $G=\mathrm{SU}(n)$ and let $(V, \rho)$ be the defining $n$-dimensional representation. Then $G$ acts transitively on $\Omega$ so $I_{\rho}$ must be a single coadjoint orbit. This is an easy example of a representation which is not convex.

Now let $G=\mathrm{SU}(2)$. The irreducible representations may be labeled by $l=0, \frac{1}{2}, 1, \frac{3}{2}, \ldots$. The representation $\left(V_{l}, \rho_{l}\right)$ acts in a space of dimension $2 l+1$. We may normalize the inner product $($,$) on \mathscr{G}^{*}$ so that when $l=\frac{1}{2}$ (the defining representation), $I_{\rho_{l}}$ is the sphere of radius $\frac{1}{2}$.

Proposition 4.1. For $l>\frac{1}{2}, I_{\rho_{l}}$ is the solid ball of radius $l$.

Proof. This follows from Theorem 3.6 and the fact that $\mathscr{G}=\operatorname{su}(2)$ has only 2 roots both of length 1 , so that all dominant integral weights are root distinct except for the one of length $\frac{1}{2}$, which corresponds to the defining representation.

We will call a representation $(V, \rho)$ balanced if $0 \in I_{\rho}$.

Proposition 4.2. Let $G=\mathrm{SU}(n)$ and $\rho$ be its defining $n$-dimensional representation. Let $\pi=\rho \wedge \rho$. Then $I_{\pi}$ is balanced if and only if $n$ is even.

Proof. Let $V$ be the space of complex $n \times n$ skew-symmetric matrices with inner product

$$
\left(M_{1}, M_{2}\right)=\operatorname{tr}\left(M_{1} M_{2}^{*}\right)
$$

Then we can define for $X \in \operatorname{su}(n)$ and $M \in V$

$$
\pi(X)(M)=X M+M X^{t}
$$

One may verify that $\pi$ is a representation of $\operatorname{su}(n)$ consisting of skew-Hermitian operators, and that its weights are just $\left\{\lambda_{i}-\lambda_{j} \mid i \neq j\right\}$ where $\lambda_{i}, i=1, \ldots, n$, 
are the entries of an $n \times n$ diagonal matrix. This then implies that $(V, \pi)$ is a realization of $\rho \wedge \rho$.

For $M \in V$ of norm 1 , and $X \in \operatorname{su}(n)$,

$$
\begin{aligned}
\Psi(M)(X) & =\frac{1}{i}(\pi(X) M, M)=\frac{1}{i} \operatorname{tr}\left(X M M^{*}+M X^{t} M^{*}\right) \\
& =\frac{2}{i} \operatorname{tr}\left(X M M^{*}\right)=2\left(X, i M^{*} M\right) .
\end{aligned}
$$

Thus $\Psi(M)=0$ if and only if the skew-Hermitian matrix $i M^{*} M$ is orthogonal to $\operatorname{su}(n)$, that is, if and only if $i M^{*} M$ is a multiple of the $n \times n$ identity matrix $I_{n}$.

It follows that if $\Psi(M)=0$ and $M$ has norm 1 then $M$ is necessarily invertible so that

$$
0 \neq \operatorname{det}(M)=\operatorname{det}\left(M^{t}\right)=(-1)^{n} \operatorname{det} M,
$$

which implies that $n$ is even.

Conversely if $n$ is even then the following is an example of a matrix $M \in V$ such that $\Psi(M)=0$ :

$$
M=\frac{1}{\sqrt{n}}\left(\begin{array}{ccccc}
0 & 1 & & & \\
-1 & 0 & & & \\
& & 0 & 1 & \\
& & -1 & 0 & \\
& & & & \ddots
\end{array}\right)
$$

As a final example, we determine the moment sets of the irreducible unitary representations of the Heisenberg group. If $\{X, Y, Z\}$ is a basis of the Lie algebra $\mathscr{G}$ with commutation relations $[X, Y]=Z$ then the coadjoint orbits are either planes

$$
\mathscr{O}_{\lambda}=\left\{f \in \mathscr{G}^{*} \mid f(Z)=\lambda\right\},
$$

for $\lambda \in \mathbb{R}, \lambda \neq 0$, or points

$$
\mathscr{O}_{x, y}=x X^{*}+y Y^{*}
$$

for $x, y \in \mathbb{R}$. The Kirillov theory associates to each of these orbits $\mathscr{O}$ an irreducible unitary representation $\rho_{\mathscr{O}}$.

Theorem 4.2. For any coadjoint orbit $\mathscr{O}$

$$
I_{\rho_{\mathcal{O}}}=\mathscr{O} \text {. }
$$

Proof. It is a well-known fact that the representation $\rho_{\mathscr{O}_{\lambda}}$ may be realized in $L^{2}(\mathbb{R})$ in such a way that $Z$ acts as the scalar $i \lambda$. Thus for any unit $C^{\infty}$ vector $v \in L^{2}(\mathbb{R}), \Psi(v)(Z)=\lambda$ so that $I_{\rho_{\theta_{\lambda}}}=\mathscr{O}_{\lambda}$. On the other hand the representation $\rho_{\mathcal{O}_{x, y}}$ is 1 -dimensional and if $v$ is a vector in the representation space then $Z \cdot v=0, X \cdot v=i x v$ and $Y \cdot v=i y v$ from which it is immediate that $I_{\rho_{\theta_{x}, y}}=\mathscr{O}_{x, y}$.

The case of a general nilpotent group has been studied in Wildberger [12]. The moment map has also been used to construct *-products on integral coadjoint orbits for compact groups (see Wildberger [13]). 


\section{REFERENCES}

1. V. I. Arnold, Mathematical methods of classical mechanics, Springer-Verlag, Berlin, 1980.

2. M. F. Atiyah, Convexity and commuting Hamiltonians, Bull. London Math. Soc. 14 (1982), $1-15$.

3. Angular momentum, convex polyhedra and algebraic geometry, Proc. Edinburgh Math. Soc. 26 (1983), 121-138.

4. V. Guillemin and S. Sternberg, Convexity properties of the moment mapping, Invent. Math. 67 (1982), 491-513.

5. _ Convexity properties of the moment mapping, II, Invent. Math. 77 (1984), 533-546.

6. S. Helgason, Groups and geometric analysis, Academic Press, Orlando, Fla., 1984.

7. A. A. Kirillov, Representations of nilpotent Lie groups, Russian Math. Surveys 17, No. 4 (1962), 53-103.

8. F. Kirwan, Convexity properties of the moment mapping, III, Invent. Math. 77 (1984), 547552.

9. B. Kostant, Quantization and unitary representations, Lecture Notes in Math., vol. 170, Springer-Verlag, Berlin, 1970, pp. 87-208.

10. On convexity, the Weyl group and the Iwasawa decomposition, Ann. Sci. École Norm. Sup. 6 (1973), 413-455.

11. J. M. Souriau, Structure des systèmes dynamiques, Dunod, Paris, 1970.

12. N. J. Wildberger, Convexity and representations of nilpotent Lie groups, Invent. Math. 98 (1989), 281-292.

13. N. J. Wildberger, On the Fourier transform of a compact semisimple Lie group, (preprint).

Department of Mathematics, University of Toronto, Toronto, Ontario, Canada M5S $1 \mathrm{~A} 1$

Current address: School of Mathematics, University of New South Wales, Kensington, NSW 2033, Australia 